\title{
The New Formula of Seismic Rigidity Method
}

\author{
Alexandr S. Aleshin \\ The Schmidt Institute of the Earth of Russian Academy of Science (IPE RAS), Moscow, Russia \\ Email: asa@ifz.ru
}

How to cite this paper: Aleshin, A.S. (2018) The New Formula of Seismic Rigidity Method. Open Journal of Civil Engineering, 8, 326-340.

https://doi.org/10.4236/ojce.2018.83025

Received: April 10, 2018

Accepted: September 24, 2018

Published: September 27, 2018

Copyright $\odot 2018$ by author and Scientific Research Publishing Inc. This work is licensed under the Creative Commons Attribution International License (CC BY 4.0).

http://creativecommons.org/licenses/by/4.0/

\begin{abstract}
This publication is a revised version of the previous article. Seismic rigidity method despite its widespread use is the object of harsh criticism from scientists who oppose it to the methodology and results of seismological registration of earthquakes and microseisms. The article substantiates the original approach based on the solution of the direct problem of seismic microzonation for the model of real soil thickness. A new formula of the seismic rigidity method is proposed, taking into account the lithological, hydrogeological and spectral features of the soil mass, as well as the position of the new seismic scale of the SSI. The formula was tested on the example of the correct description of the features of macroseismic effects on the territory of Leninakan at the Spitak earthquake in 1988. Linear estimates according to the formula of seismic rigidity in the seismic microzoning area represent changes in seismic intensity in the most contrast way. It is shown that the real estimates of seismic intensity under strong seismic effects (by I > VII degree) will not exceed those given by the formula of the seismic rigidity method.
\end{abstract}

\section{Keywords}

Seismic Microzonation, Degree of Intensity, Seismic Rigidity Method, Ground Category, Referent Ground, Seismic Impacts, Spectral Characteristics, Dynamics Coefficient, Model, Continuity

\section{Introduction}

The seismic rigidity method (SRM) is historically one of the first instrumental methods of assessing the impact of soil properties on the parameters of seismic effects, which currently causes a contradictory attitude. On the one hand, it is the most frequently used method of seismic microzonation (SMZ); on the other hand, it is hardly possible to find in the arsenal of SMZ methods another method that is subjected to such fierce and diverse criticism. It should be noted that this criticism is not presented in the form of printed articles, but constantly arises in 
various discussions, in private opinions, etc., which only complicates public discussion. What to be considered and what to reject? This article attempts to understand this interesting and important from the practice point of view question. This publication is a revised version of the previous article [1].

\section{Historical Background}

For the quantitative ratios, linking the soil properties with changes in seismic intensity S. V. Medvedev [2] was used the dependence of seismic intensity from three factors: the value of seismic rigidity-the product of elastic wave velocity on density, groundwater level and resonance phenomena in the thickness of the soil. Formally, this dependence is expressed by the relation: $\Delta I_{\Sigma}=\Delta I_{S}+\Delta I_{W}+\Delta I_{R}$, where consistently spelled out the above-mentioned factors. Consider each of the factors in more detail.

\subsection{Accounting for Seismic Rigidity}

S. V. Medvedev used the seismic rigidity as the results of seismic measurements of longitudinal wave velocities of the corresponding types of soils. The density values of the respective types of soils were taken from engineering-geological surveys data. The obtained values of seismic rigidity of different types soils were compared in relation to the values of the highest seismic rigidity corresponding to granites. Then a functional dependence was found, which best satisfied the results of macro-seismic definitions, linking the increment of the intensity with the soil parameters. Can be only be surprised (and admire!) as on the basis of such simple assumptions of S. V. Medvedev the expression determining increase of seismic intensity on parameters of soil in the form of the following expression is received: $\Delta I_{S}=1.67 \cdot \lg \left(\frac{\rho_{0} V_{0}}{\rho_{i} V_{i}}\right)$.

At that the proportionality factor $B=1.67$ was obtained as an arithmetic mean of several partial definitions. No physical justification of the obtained coefficient was given. Only subsequently, there were physical considerations for explaining the derivation of the resulting coefficient on the basis of assumptions about the equality of flows of seismic energy in the two neighboring points with different soil properties.

Let's check the validity of this assumption. The expression for the density of energy flow can be written in the following form: $W=U^{2} /\left(\rho V_{s}\right)=\left(\rho V_{s}\right) \cdot \dot{Z}^{2}$, where $\rho, V_{s}$ is the density of ground and velocity of elastic waves in the medium, $\dot{Z}, U$ is the vibrational velocity and stress in the elastic wave. Assume that the densities of energy flow at two neighboring points are equal. This means: $W_{0}=W_{1}$ or $\left[\left(\rho V_{s}\right) \cdot \dot{Z}^{2}\right]_{0}=\left[\left(\rho V_{s}\right) \cdot \dot{Z}^{2}\right]_{1}$. Assume further that, according to the seismic scale, the change of seismic intensity on 1 point corresponds to a doubling of the vibrational velocity. This can be expressed by the following formula: $\dot{Z}_{1} / \dot{Z}_{0}=2^{\Delta I}$. Taking of logarithm of this expression and expressing the ratio of the vibration velocities through the ratio of seismic rigidity in accordance with 
the above relations, we obtain $\Delta I \cdot 0.3=0.5 \cdot \lg \left[\left(\rho V_{s}\right)_{0} /\left(\rho V_{s}\right)_{1}\right]$ or finally: $\Delta I=1.67 \cdot \lg \left[\left(\rho V_{s}\right)_{0} /\left(\rho V_{s}\right)_{1}\right]$.

Thus, the first and the main member of Medvedev's formula was obtained, which determined the increment of the intensity on the soils. Name of the first member of dependence have become the name of the seismic rigidities method (SRM) as whole.

\subsection{The Effect of Water Saturation}

The second term in Medvedev's formula determines the dependence of the intensity from the groundwater level. The macroseismic data of strong earthquakes showed that the high groundwater level corresponds to the increment of seismic intensity on 1 point. The relevant macroseismic data obtained in sandy loam, loam and fine sand may presented in the form of "simplest" dependence: $\Delta I_{w}=\exp \left(-0.04 h^{2}\right)$, where $h$ is the depth of the groundwater level.

Subsequently, before the exponent appeared lithological factor $K$. At that, according to [3], the coefficient $K$ must be equal to:

1-for sandy soils, plastic and fluid sandy loam, smooth, and flowing loams and clays;

0.5-solid loam, solid, semi-solid and hard-plasticity loam and clay, coarse grounds with sand and clay filler content of at least 30\% and highly weathered rock;

0 -for dense coarse-grained grounds from igneous rocks with the content of sand and clay filler up to $30 \%$ and a small crust of weathering and other soil.

\subsection{On the Thickness of the Active Layer}

The macro-seismic data, formed the basis of the seismic rigidity method, showed that seismic intensity increment is determined by the properties of the soil thickness of not more than $10 \mathrm{~m}$. This empirical result was not explained in the initial publications on the SMZ. In document [3] "the thickness of the calculated thickness is taken equal to $10 \mathrm{~m}$, counting from the planning mark, or other reasonable, but not more than $20 \mathrm{~m}$ ". Finally, in the last standard [4], probably under the influence of American norms [5], the calculated power is determined by the value of 30 meters relative to the planning mark. It is superfluous to remind that the change in the calculated thickness in the general case changes the increment of seismic intensity.

\subsection{Resonance Effects}

Accounting of resonant phenomena in the soil thickness is the least developed part of the seismic rigidity method. The influence of resonance effects in a number of normative documents [6] is taken into account by means of the simplest model-a layer of soft soils with the shear waves velocity $V_{1}$, seismic rigidity $R$ and thickness $h$, lying on half-space with shear wave velocity $V_{0}$ and seismic rigidity $R_{0}$. This model is described by relations, one of which $f=V / 4 h$ deter- 
mines the resonance frequency $f$, and the second $-R_{0} / R$ controls the maximum value in the resonance. The higher contrast of rigidity of layer and half-space, the more resonant the frequency response graph is.

It should be note that the accounting of resonance phenomena with the help of a homogeneous ground massif model laying on a rigid half-space was incorporated into seismic norms in the precomputer age. How to produce the calculations with the use of computer technology in the case of more complex models of the soil massif, in this rule does not say anything.

\subsection{Medium Ground Conditions}

The concept of "medium" soils is one of the key in normative documents of Russia used till now. Medium ground conditions are the same as "medium" soils is according to rules [4] are soils of the II seismic category. It is in this form that this concept was used by the drafters of the map of General Seismic Zoning of GSZ-97 [7]. It is worth paying attention to the fact that "medium" soils include such a variety of properties of dispersed soils that one this excludes the possibility of its practical use for the purposes of GSZ. Indeed, according to the latest version of Table 1 from SP 14.13330, the properties of soils of the II category are characterized by seismic rigidity in the range $350-1500\left(\mathrm{~g} / \mathrm{cm}^{3} \cdot \mathrm{m} / \mathrm{s}\right)$, which by the seismic rigidity method and the MSK-64 scale is equal to a change in the increment of seismic intensity of about 1 point. It is to this diapason the "medium" soils determining the initial seismic intensity on the GSZ maps, can change "legally". And this inevitably leads to poorly controlled errors in the appointment of the initial seismic intensity on the maps of the GSZ.

Apparently, aware of the inadmissibility of such a broad understanding of the "medium" soil conditions, the authors of the standard [3] narrowed the field of "medium" soil, defining their parameters $V_{s}=250-350 \mathrm{~m} / \mathrm{s} ; \rho=1.7-1.8 \mathrm{~g} / \mathrm{cm}^{3}$. At the same time without any reservations these soils are defined as soils of the II category. It is not clear how the authors of this standard did not pay attention to the fact that in this definition sharply narrows the field of soils of the II category. The properties of the part are identified with the properties of the whole.

We draw attention to another inaccuracy of the standard [3]. Along with the useful narrowing of the field of parameters for "medium" soils, the authors of the document considered it necessary to accept as one of the most important tasks of the SMZ "the choice of reference (medium) soil", without explaining at the same time how to act if the parameters of the "medium" soil do not coincide with the above "narrowed" parameters.

\subsection{Criticism of the Method}

The critical attitude to the method of seismic rigidity, perhaps, most clearly manifested itself in the opposition to it other instrumental methods of the SMZ. According to some scientists [8], the method of seismic rigidity for SMZ is an indirect calculation method for assessing variations of seismic intensity on the 
site. In contrast, this in favor of engineering-seismological methods of SMZ says factors such as large depth and relatively low frequencies, characteristic of seismic vibrations in earthquakes.

Another serious drawback of the seismic rigidity method is considered by the opponents of the method to be the lack of spectral estimates.

Finally, the critics of the seismic rigidity method consider the use of linear representations for the connection of deformations and stresses to be another disadvantage, while in seismic vibrations at strong earthquakes nonlinear processes are essential.

\section{Modernization of Seismic Rigidity Method}

In our point of view, the criticisms levelled at the SRM, despite many equitable provisions, do not relate to the main difference between the SRM and other methods of the SMZ. Namely, the parameters of seismic vibrations are determined exclusively by the properties of the geological environment, or rather by the magnitude of seismic rigidity. Without knowing the properties of the soil massif, it is impossible to determine the parameters of seismic vibrations. All other methods of SMZ - seismological registration of earthquakes or microseisms-claim to independent determination of seismic parameters without taking into account the properties of the seismic environment, which, in our opinion, is an illusion, because, not knowing the properties of the medium, it is impossible to separate the properties of the incoming seismic impact and response to it of properties of the medium. On the contrary, knowledge of the properties of the medium allows to determine the reaction of the soil massif to the input seismic action by solving a direct problem. Without knowledge of the properties of the medium, the response of the system of soil layers to the incoming seismic impact is ambiguous. After these necessary remarks, we will continue the critical analysis of seismic rigidity method, noting its disadvantages and introducing the correct ratios. Let's start with the basic formula of the SRM. Its presentation in normative document [3] is incorrect.

\subsection{Seismic Rigidity}

The first base member of the formula is incorrect. The premise from which the ratio was derived is also incorrect: the equality of the power flow in two neighboring points with different soil structure. This has already been shown in previous work [9], we will now repeat this conclusion. Let's start by checking the assumption about the equality of energy flows at close points on the rock and soft soil. Let's make quantitative estimates. The power flux density $\mathrm{W}$ in a plane elastic wave is [10]

$$
W=\frac{U^{2}}{\rho V_{s}}=\rho V_{s} \cdot \dot{Z}^{2}
$$

where $U$ and $\dot{Z}$ are respectively stress and oscillatory velocity. 
In Figure 1, there are two of the simplest models of the soil-outcrop on the day surface of the rock and soft soil layer underlying by rock foundation. Model parameters are shown in Figure 1, taken from the normative document [3]. Soft soil is represented by soils of the II category, and rock soil belongs to the I category. Seismic rigidity of rock $R_{0}=\rho_{0} V_{s 0}$ and soft soil $R_{1}=\rho_{1} V_{s 1}$ in comparative units (without specifying a dimension) is equal to 2.0 and 0.5 , respectively. We also assume that the thickness of soft layer is that may not take into account the resonance phenomena. From the elastic half-space normally falls the plane elastic shear wave unit stress $U_{0}=1$. For the rock model, by substitution into the above expression of the energy flux density, we obtain $W_{0}=0.5$. For a model with soft soil during the transition from rock to a upper layer of soft soil, the stress is expressed by the formula $U_{1}=U_{0} \cdot \frac{2 R_{1}}{R_{0}+R_{1}}$.

On substitution into this expression of the parameters soft soil get $U_{1} \cong 0.4$. Further, using the expression for the power flux density, $W_{1}=\left(U_{1}\right)^{2} /\left(\rho V_{s}\right)_{1}$ we obtain by substituting the corresponding parameters $W_{1}=\left(0.4^{2} / 0.5\right)=0.32$. Thus, the power flux density in soft soils is more than 1.5 times less than on the rock. This phenomenon is explained by the fact that during the transition from the rock to the soft soil, most of the energy is reflected back into the half-space.

Despite the fact that the energy density on the soft soil is much lower than in the rocky, the amplitude of the displacement rate (as well as accelerations) on the soft soils is greater than on the rock. The amplitude of displacement rate of the $\dot{Z}$ at the free surface will be determined by the expression $\dot{Z}_{0}=2 U_{0} / R_{0}=2 / 2.0=1$. The stress in the elastic wave propagating in the soft soil, as shown above, is $U_{1}=$ 0.4. Hence, the displacement rate of the free surface of the soft soil, taking into account the doubling will be $\dot{Z}_{1}=2 U_{1} / R_{1}=0.4 \times 2 / 0.5=1.6$. Thus, the amplitude of the displacement rate on soft soil is 1.6 times higher than the displacement rate on rocky soil. Both results are of fundamental importance. On the one hand, the condition of equality of the energy flux density on soft and rocky soils is not observed and, therefore, the formula of seismic rigidity method is incorrect.
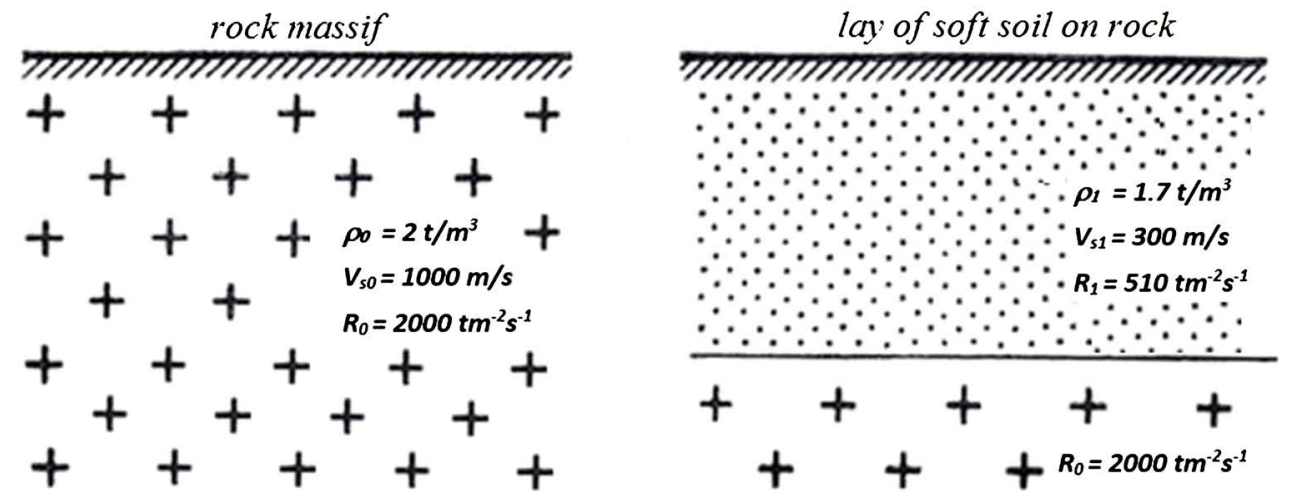

Figure 1. The model examples of calculations. 
On the other hand, although the amplitude of displacement rate (and accelerations) on the soft soil is noticeably greater than on the rocky one, at any ratio of seismic rigidity of the soft dispersive and rocky soil, the increase in the amplitude of displacement rate (and accelerations) will not exceed 2 times. Indeed, $\dot{Z}_{0}=2 U_{0} / R_{0}, \dot{Z}_{1}=2 T U_{0} / R_{1}$, where $T=2 R_{1} /\left(R_{1}+R_{0}\right)$. From here it is easy to get $\dot{Z}_{1} / \dot{Z}_{0}=2 /\left(\left(R_{1} / R_{0}\right)+1\right)$.

If $R_{1} / R_{0}<1$ (soft soil on a rigid base), the ratio of displacement rates will be within the interval $(1 ; 2)$. If more rigid soil covers less rigid one $R_{1} / R_{0}>1$, the ratio of the displacement rate lies in the range of the segment $(0 ; 1)$. In this case, the ratio of displacement rate can be higher than 2 . Thus, we have obtained a general result: due to seismic rigidity, the change in displacement rates (and accelerations) can be different depending on the ratio of acoustic rigidities of the soil layers that make up the cut, but not twice, as is currently accepted in regulatory documents. This casts doubt on the correctness of the use of Table 1 of the standard [3] and methods of evaluation of the increment of seismic intensity on measurements of the amplitudes of the recordings of weak earthquakes. However, this result does not contradict the macroseismic data, assuming that on the change in displacement rates, in addition to seismic rigidity, contribute resonance effects.

The condition of equality of the energy fluxes at the neighboring points at the front of a seismic wave is maintained to a level at which the properties of the medium in the two adjacent (from the point of view SMZ) points do not change. In geological terms, this level usually corresponds to the roof of rocky soil. Above this level, the rocky soil is covered with a soft sedimentary cover, the properties of which are quite remarkable (compared with the underlying thicknesses) vary both in depth and laterals. For correct calculation of frequency characteristics of a soil massif it is better to accept a sole of a layer of soft soils for this level, and to count frequency characteristic as the relation of the spectra of oscillations registered in some point of a surface of soft soils to the similar spectrum on an output on a day surface of rocky soils. However, it is not important the presence of the rock. It is possible to imagine a case where the condition of constancy of the properties of the medium is made at a different level (the level of reduction) inside the array of soft soil. Then it is appropriate to determine the frequency characteristic of the soil massif in relation to the recording of oscillations or at the inner point of the medium at the reduction level (in this case, it must take in mind that there is no usual for the day surface of the amplitude doubling!), or at the point of exit of this level on a day surface.

The fact that the energy in the elastic wave on the rock is greater than on soft soils does not contradict the experimentally observed facts of large destruction on weak soils. Simply in this case, most of the total energy is transferred to the structure, i.e. there is a better coordination of soil properties and structure. For the same reason massive heavy constructions placed on stony soil had to be subjected to more considerable damages that it was noted by the known Japanese 
scientist K. Suehiro [11]. There is one more circumstance which should be considered at determination of the correct ratio between amplitudes of seismic acceleration and parameters of the soil massif. It is necessary to use a new seismic scale [12], in which the change of intensity by 1 point corresponds to the change of accelerations by 2.5 times. All the above considerations allow us to present the relationship between the intensity of seismic vibrations with the seismic rigidity of the soil massif in the form of a modified seismic rigidity formula:

$$
\Delta I_{S}=2.5 \lg \left[2 /\left(R_{1} / R_{0}+1\right)\right]=0.75-2.5 \cdot \lg \left(R_{1} / R_{0}+1\right),
$$

where indices 0 and 1 refer respectively to the lower and upper layers of the soil.

When $R_{0} \gg R_{1}$-hard rock base covered with a layer of soft soil- $\Delta I_{S}=0.75$ points.

At $R_{0}=R_{1}$-the properties of layers do not differ $-\Delta I_{S}=0$ points.

When $R_{1}=2 R_{0}$-the upper layer is 2 times more rigid than the lower $\Delta I_{S}=$ -0.45 points. Thus, the total range of seismic rigidities influence on seismic intensity increment is about 1.2 points. Fundamentally important is the definition of "zero" increments of seismic intensity by increased values of seismic rigidity $R_{0}=2.5 \mathrm{~g} / \mathrm{cm}^{3} \cdot 800 \mathrm{~m} / \mathrm{s}=2000\left(\mathrm{~g} / \mathrm{cm}^{3} \cdot \mathrm{m} / \mathrm{s}\right)$, which eliminates the change in their properties (in first the shear wave velocity) under strong (more than VII points) seismic effects, on that marked in [13].

\subsection{The Calculated Thickness}

Soil typically represents a layered medium, layer properties which differ sometimes quite significantly. Because the seismic properties of the layered medium can be determine by the average values of the densities $\rho_{a v}$ and average velocities of the shear waves $V_{a v}$ defined by the formulas:

$$
\rho_{a v}=\Sigma\left(\rho_{i} \cdot h_{i}\right) / \Sigma h_{i} ; V_{a v}=\Sigma h_{i} / \Sigma\left(h_{i} / V_{i}\right) ; R_{a v}=\rho_{a v} \cdot V_{a v}
$$

The thickness of the layer having an effect on the amplitude of vibrations on the day surface, should no less than a quarter of the wavelength of the shortest component of seismic vibrations. In other words, $h \geq 0.25 \lambda=0.25 V_{s} / 10 \mathrm{~Hz}$. For $V_{s}=300 \mathrm{~m} / \mathrm{s} h \approx 7.5 \mathrm{~m}$. For $V_{s}=1000 \mathrm{~m} / \mathrm{s} h \approx 25 \mathrm{~m}$. The validity of these estimates illustrates Figure 2, on which presents the results of modeling the dynamic coefficients of the layer model with the parameters $V_{s}=270 \mathrm{~m} / \mathrm{s}, \rho=1.7$ $\mathrm{g} / \mathrm{cm}^{3}$, lying on the half-space with the parameters $V_{s}=1000 \mathrm{~m} / \mathrm{s}, \rho=2.2 \mathrm{~g} / \mathrm{cm}^{3}$. It is obvious that the maximum of every curves are kept at a constant level up to $h \approx 7.5 \mathrm{~m}$, which corresponds to a wavelength of $30 \mathrm{~m}$ for the highest-velocity soil $V_{s}=1000 \mathrm{~m} / \mathrm{s}$. The wavelength at $f=10 \mathrm{~Hz}$ is $100 \mathrm{~m}$. This allows to estimate the thickness of the soil of the order of $25 \mathrm{~m}$, which closely corresponds to the normative assessment $h=30 \mathrm{~m}$. The previously received the calculated thickness of the soil $h=10 \mathrm{~m}$ is insufficient for reliable assessment of the properties of the soft soils, since it is less than a quarter of the wavelength and it is influenced by the properties of the underlying medium. 


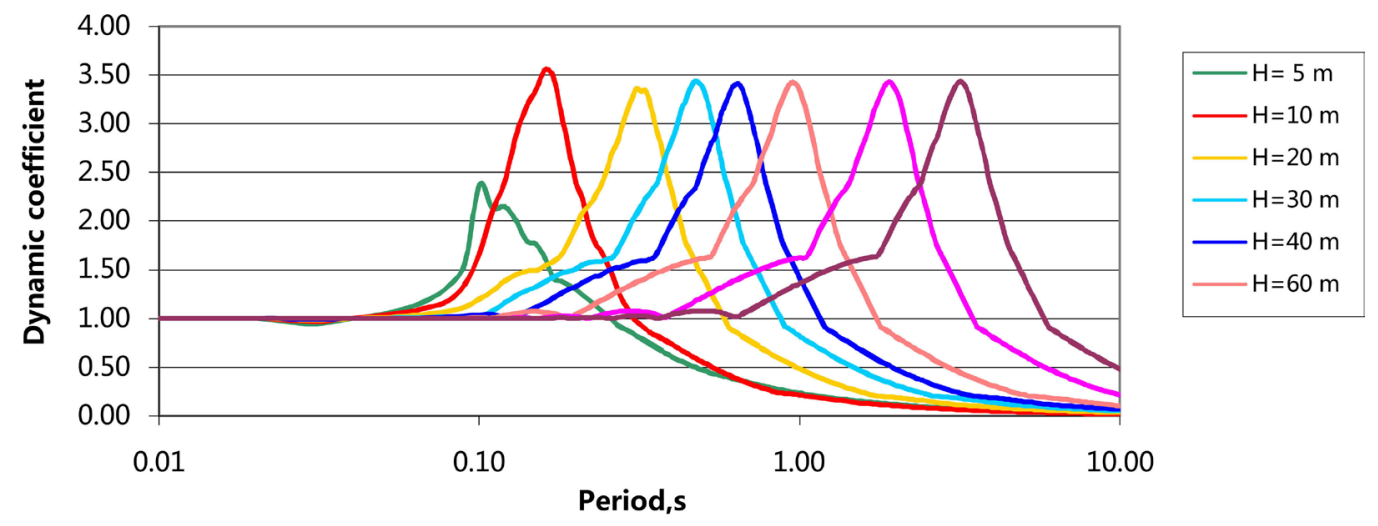

Figure 2. The series of graphs of the dynamic coefficients of the layers varying thickness.

\subsection{The Effect of Hydration}

Changing the properties of the soil under the influence of various factors, including water saturation, is taken into account by a change in seismic rigidity. Water saturation increases the density of the soil and affects the velocity of shear waves in different ways due to changes in contact adhesion at grain boundaries. The influence of water saturation on the seismic properties of fine-grained soils was considered in the recent paper [14]. In some cases (for example, when soaking loess) reduction of contact strength at grain boundaries can lead to partial or complete loss of bearing capacity of the soil. Seismic effects of this phenomenon were studied in a number of papers [15] and therefore there is no need to repeat the results here. No other effects (such as hydraulic shock, as indicated in the early works on this problem [2]) due to low stress is not examined and therefore there is no need to introduce an additional member, taking into account the influence of water saturation on the increment of seismic intensity.

\subsection{Spectral Features}

Now consider the question of taking into account the spectral characteristics of the soil massif by seismic rigidity method. Formally, the spectral features of the soil reaction to seismic effects in the seismic rigidity method are taken into account by the $\Delta I_{R}$ member. Our goal is to develop a clear procedure for taking into account the spectral characteristics of the soil. For this purpose, the results of computer simulation of a series of soil conditions models are considered. The general model is a 30-meter layer of soil located on an elastic half-space with parameters $V_{s}=1000 \mathrm{~m} / \mathrm{s}, \rho=2.2 \mathrm{t} / \mathrm{m}^{3}$. The parameters of the upper layer of the soil are changed sequentially, respectively, with the parameters of the soil categories from A to E. Elastic vibrations were excited by a short pulse with an amplitude of $0.1 \mathrm{~g}$, which ensures the linearity of the "stress-strain" relations. The simulation results given in [16] showed that the dynamic coefficient adequately reacts to the properties of the soil massif. In a sufficiently wide range of seismic rigidity changes, the maximum dynamic coefficient $b$ is in the range of values from 2 to 6 or more, that is, it changes at least 3 times. In the mentioned work 
[16] is determined the dependence of the maximum dynamic factor $b$ from seismic rigidity $R$. Thus, the spectral properties of the soil massif can be described without the use of the concept of soil categories. The maximum of dynamic coefficient is functionally related from the seismic rigidity of the soil massif.

If it is assume that the value $b=2$ corresponds to the increment of seismic intensity $\Delta I=0$ (in other words, the absence of resonant phenomena), the increment of seismic intensity due to the spectral properties of the soil massif can be determined using the expression: $(b / 2)=2.5^{\Delta I}$. Here the coefficient 2.5 corresponds to the data of new seismic scale [12].

Taking logarithm, we obtain the expression $\Delta I_{R}=2.5 \lg b-0.75$.

At $b=2, \Delta I_{R}=0$; at $b=4, \Delta I_{R}=0.75$; at $b=6, \Delta I_{R}=1.2$ points.

By combining expressions $\Delta I_{S}$ and $\Delta I_{R}$, you can finally get:

$$
\Delta I_{\Sigma}=0.75-2.5 \cdot \lg \left(R_{1} / R_{0}+1\right)+2.5 \lg b-0.75=2.5 \lg b R_{0} /\left(R_{0}+R_{1}\right) .
$$

This is the main formula of SRM. In this expression it takes into account the influence of seismic rigidity, water saturation (through the influence of water content on $V_{s}$ ) and the spectral characteristics of the soil massif.

\subsection{Accounting of Non-Linearity}

Given relations are true in the linear range of the ratios, "stress-strain" during acceleration in dispersive soils is not more than $0.2 \mathrm{~g}$. Herewith nonlinear phenomena are not taken into account. Why do we need these ratios, when we consider that the most important assessments for the SMZ take place with strong movements of the soil at intensities above VII-VIII degrees? The formula of seismic rigidity method is important for evaluation of seismic effects as upper assessment. It means that the realistic assessment of seismic effects under strong seismic impacts (more than VII points) will not exceed the one given by formula of the seismic rigidity method (see [5] p. 22 and Figure C 3.3.2-5).

Note that as the microseismic technique, so registration and processing weak (less than $\mathrm{V}$ points) of seismic vibrations from earthquakes are not suitable for a correct account of the nonlinear phenomena under strong seismic oscillations.

\section{Example of Practical Application of the Formula SRM}

Here is given a practically important example of the use of the derived formula SRM, which explains all the features of the developed methodology. Three considered model examples relate to the interpretation of the situation that took place in Leninakan in a large Spitak earthquake of 1988. The parameters of the models from data of [17] are shown in Table 1. Layers and their parameters are exactly the same except the thickness of the upper layer represented by rigid tuffs.

The results of calculations in the form of graphs of dynamic coefficient for three models of soil thickness are shown in Figures 3-5. Let us explain that 
Table 1. The parameters of soil thickness models.

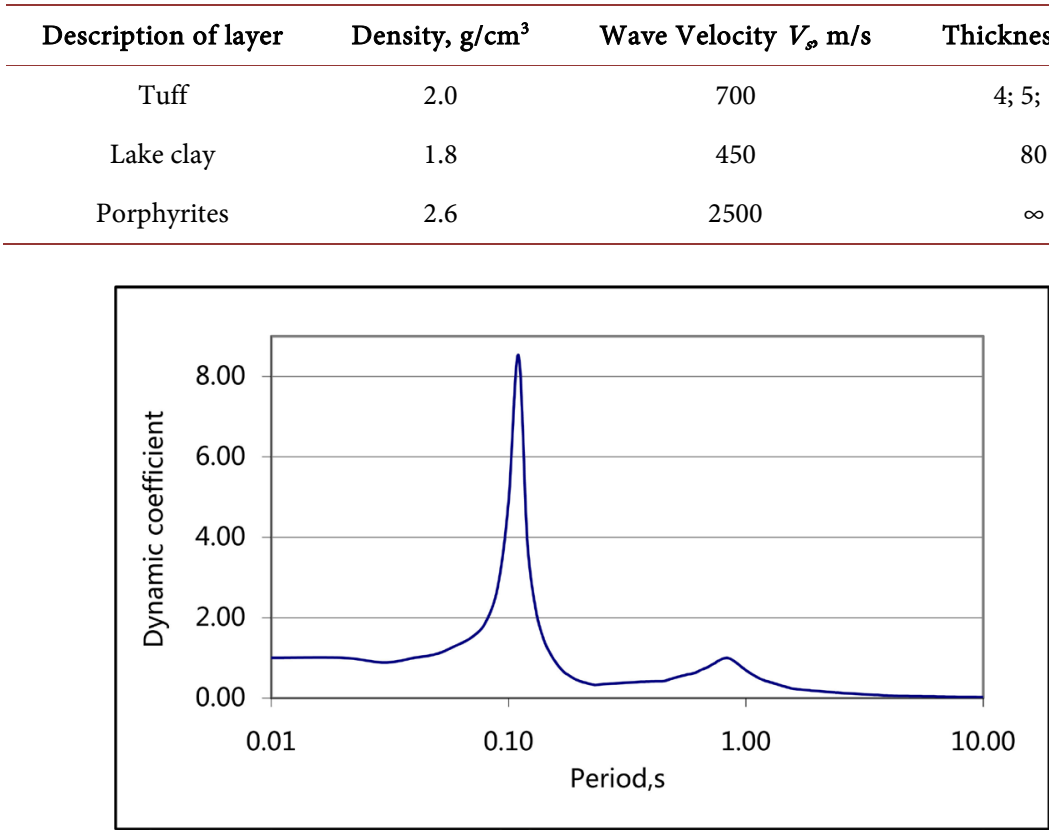

Figure 3. The dynamic coefficient, model $1, h=4 \mathrm{~m}$.

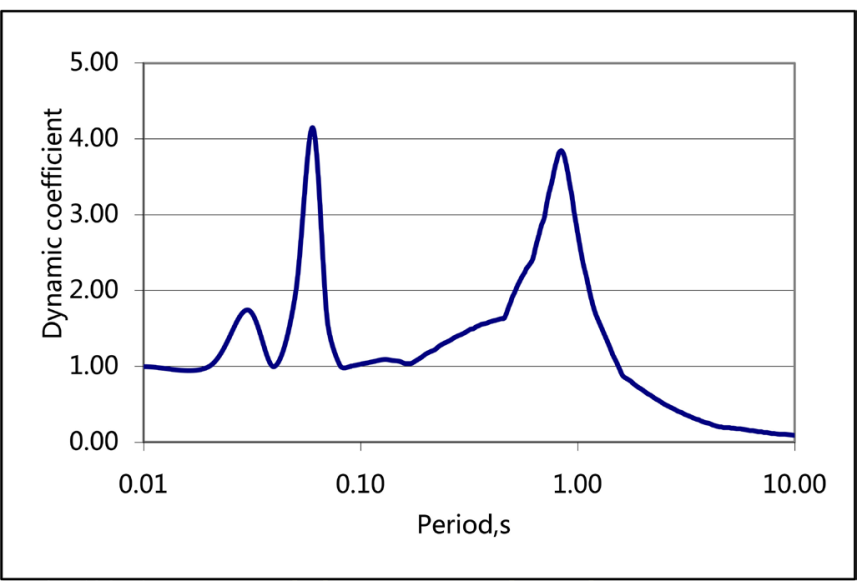

Figure 4. The dynamic coefficient, model $2, h=5 \mathrm{~m}$.

models differ only in the thickness $h$ of the upper layer of rigid tuffs. Figure 3 shows the case of a sharp resonance of the top layer for a short period approximately $0.1 \mathrm{~s}$ or at frequencies of about $10 \mathrm{~Hz}$.

The maximum of the dynamic coefficient $b=8$. Seismic rigidity of the upper 30-meter layer is equal to $R_{1}=827\left(\mathrm{tm}^{-2} \mathrm{~s}^{-1}\right)$, seismic rigidity of the rock half-space $R_{0}=6500\left(\mathrm{tm}^{-2} \mathrm{~s}^{-1}\right)$. Hence, the Formula (3) can be obtained $\Delta I=2$ points in the period range $0.1-0.12 \mathrm{~s}$ or frequencies $8-10 \mathrm{~Hz}$. The width of the spectral peaks is determined at 0.5 of the maximum.

For the model 2 with a layer thickness of tuff $h=5 \mathrm{~m}$, we have a graph of the dynamic coefficient, presented in Figure 4. This chart has two peaks at high and low frequencies: $b_{1}=4.1$ for period with about $0.06 \mathrm{~s}$ and $b_{2}=3.8$ for the 


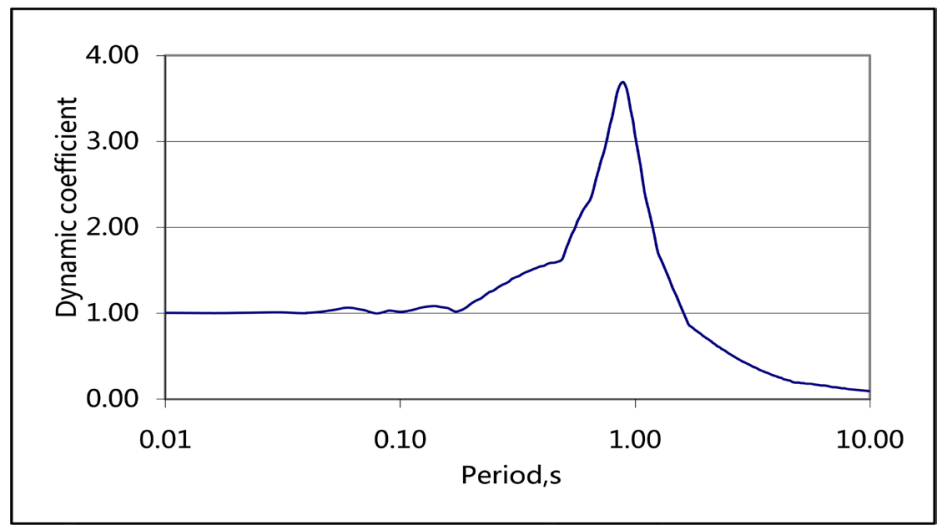

Figure 5. The dynamic coefficient, model $3, h=10 \mathrm{~m}$.

period of $0.86 \mathrm{~s}$.

The increment of seismic intensity in the maxima the dynamic coefficients are respectively equal to $\Delta I_{1}=1.4$ and $\Delta I_{2}=1.3$.

Finally, for the model with the tuff thickness $h=10 \mathrm{~m}$, the dynamism coefficient graph is shown in Figure 5. Type of chart is completely different: the maximum of the dynamic coefficient $b=3.6$, concentrating in the range of 0.53 to $1.25 \mathrm{~s}$ and frequencies of 0.8 and $1.9 \mathrm{~Hz}$.

Seismic rigidity of the upper 30-meter layer in this case is $642\left(\mathrm{tm}^{-2} \mathrm{~s}^{-1}\right)$. At the same time, by the formula SRM we obtain:

$\Delta I_{\Sigma}=2.5 \lg (3.6 \times 6500 /(6500+642))=1.3$ degrees in the frequency range of about $1 \mathrm{~Hz}$.

A few comments should be made on the results.

1) The increments $\Delta I_{\Sigma}$ by the formula SRM are determined relative to the values of seismic intensity on hard rocky soils.

2) The formula SRM takes into account the effect of seismic rigidity, water saturation, and resonance effects.

3) The formula SRM allows obtaining increments of seismic intensity with an accuracy of tenths of a point.

4) Thus, the total seismic intensity is summa of increment $\Delta I$ and values of the initial seismic intensity. The accuracy of determination both values are up to 0.1 points. The general averaging is performed at the final stage of calculations.

It must take attention to the fact that the values of the dynamic coefficients depend even on small changes in the thickness of the upper layer of the soil (Compare the dynamic coefficients for the model $1 h=4 \mathrm{~m}$ and the model $2 h=$ $5 \mathrm{~m})$.

Now let's consider how the deep features of the soil structure affect the spectral properties of ground massif. Figure 6 illustrated the serial curves of dynamic coefficients for different depths of the rock foundation.

Despite the large range of changes in the depth of the rock base, the general appearance of the graphs of the dynamic coefficients does not change. There are the same two humps of spectral characteristics, the approximate equality of the 


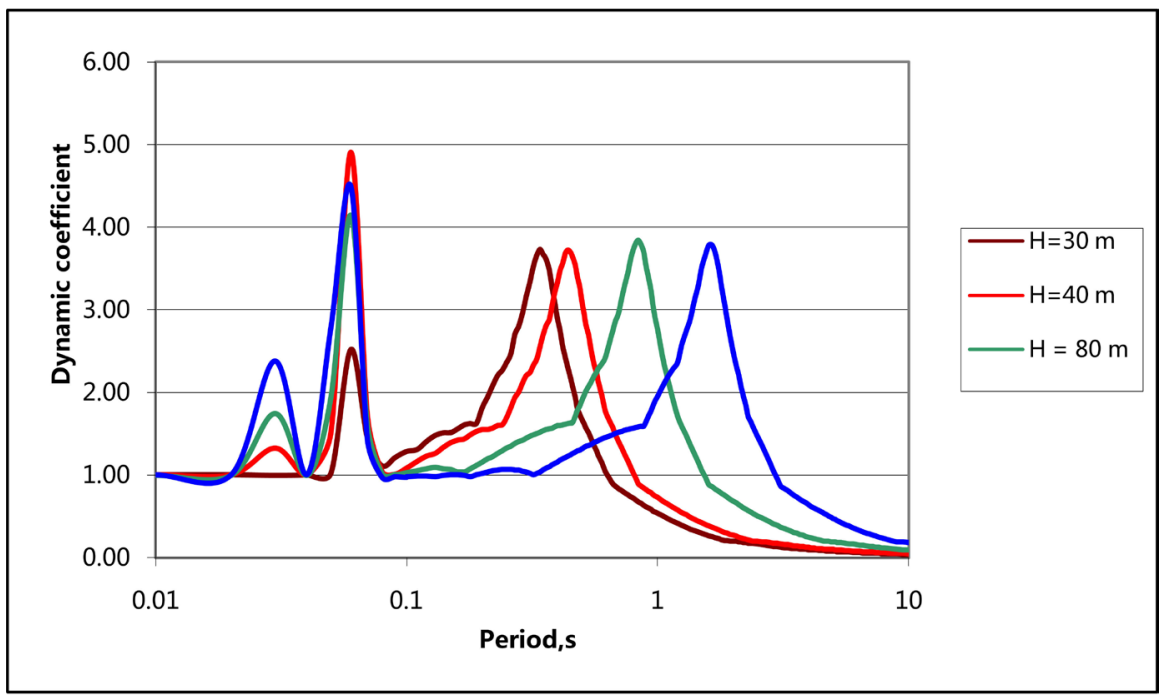

Figure 6. The series of dynamic coefficients graphs depending on the depth of the rock base $h$.

width of spectral peaks are conserving. The frequency of the low-frequency spectral peak $\mathrm{F}$ varies naturally according to the ratio: $f=V_{s} / 4 h$, where $h$ is the depth of the rock base.

The observed features of the spectral characteristics allow us to assert that the shape of the spectral characteristics in the high-frequency part of the spectrum is determined by the features of the upper part of the section by a depth no more than $30 \mathrm{~m}$. To assess the spectral characteristics of seismic vibrations in the low-frequency range, it is necessary to know peculiarities of the geological structure to a large depth.

If the data on the structure of the section to the depths of about $80 \mathrm{~m}$ were taken into account when carrying out the SMZ in Leninakan, it would be possible to calculate the frequency characteristic in advance (similar to the graph Figure 6 red curve) and correctly calculate the seismic loads on 12-storey buildings.

\section{Conclusions}

1) The concept of the engineering-geological model is the base of the method of seismic rigidities.

2) The drawbacks of the previous method and calculation formula of SRM include:

a) the assumption of equation of fluxes seismic energy at the neighboring points, which differ in the engineering-geological structure of the soil massif;

b) taking into account the influence of water content with the use of a special member in the formula of SRM;

c) the lack of clear recommendations regarding calculation of the spectral characteristics of the soil massif;

d) use in a calculation formula of the outdated notions of the MSK-64 scale. 
3) The formula of SRM eliminating the noted disadvantages and taking into account lithological, hydrogeological and spectral features of the soil massif, is proposed. The formula takes into account the position of the new seismic scale.

4) The formula is tested on the example of a description of the macroseismic effects in the territory of Leninakan during the Spitak earthquake in 1988.

5) It is shown that the main contribution to the parameters of seismic vibrations is made by the upper part of the section with thickness not more than $30 \mathrm{~m}$.

6) The method of seismic rigidity at the site of seismic zoning represents a most contrasting way of evaluation of seismic dangerous. Linear estimates allow to allocate the most dangerous in the seismic relation sites. Nonlinear processes in general smooth out the observed variations. Realistic assessment of seismic intensity with strong seismic load (I > VII points) do not exceed those that are given formula of the method of seismic rigidities.

\section{Conflicts of Interest}

The author declares no conflicts of interest regarding the publication of this paper.

\section{References}

[1] Aleshin, A.S. (2016) Apologya metoda seismicheskih skorostey [Apology of Seismic Rigidity Method]. Earthquake Engineering, Safety of Structures, No. 3, 13-21. (In Russian)

[2] Medvedev, S.V. (1962) Engineering Seismology. Akademia Nauk Press, Moscow.

[3] (1986) Respublikanskie stroitel'nye normy. Stroiizda, Moscow. (In Russian)

[4] (2014) Svod pravil. Stroitel'stvo v seismicheskikh rayonakh. FAU FCS Publication, Moscow. (In Russian)

[5] BSSC (Building Seismic Safety Council) (2003) NEHRP Recommended Provisions for Seismic Regulations for New Buildings and Other Structures. Part 1: Provisions, Prepared by the Building Seismic Safety Council for the Federal Emergency Management Agency (Report FEMA 450), Washington DC, 356 p.

[6] (1973) Recommendations on Seismic Zoning (RSM-73). Voprosy Inzhenernoj Seismologii, No.15, 6-34. (In Russian)

[7] Ulomov, V.I. and Shumilina, L.S. (1998) Komplekt kart obtshego seismicheskogo rayonirovaniya territorii Rossiyskoy Federatsii [Set of Maps of General Seismic Zoning of Territory of the Russian Federation]. Earthquake Engineering, Safety of Structures, No. 4, 30-34. (In Russian)

[8] (2016) Metodicheckoe posobie Rukovodstvo po uchetu seismicheskikh vozdeistviji pri proektirovanii gidrotekhnicheskikh sooruzheniji [Manual for Accounting of Seismic Effects in the Design of Hydraulic Structures]. FAU FCS Publication, Moscow. (In Russian)

[9] Aleshin, A.S. (2010) Seismicheskoye mikrorayonirovaniye osobo otvetstvennych obyectov [The Seismic Microzonation of the High Important Objects]. Svetoch Plus Publication, Moscow, 304 p. (In Russian)

[10] Isacovich, M.A. (1973) Obtschaya akustika (General Acoustics). Nauka, Moscow, 
496 p. (In Russian)

[11] Suehiro, K. (1935) Injenernaya seismologiya (Engineering Seismology). Economicheskaya Zhizn, Moscow, 167 p. (In Russian)

[12] Aptikaev, F.F. (2012) Instrumental'naya shkala seismicheskoy intensivnosti [The Instrumental Seismic Intensity Scale]. Nauka i obrazovaniye, Moscow, 76 p. (In Russian)

[13] Gusev, A.A. (2002) O seismologicheskoy osnove norm seismostoykogo stroitel'stva v Rossiyi [On the Basis of Seismological Standards of Earthquake-Resistant Construction in Russia]. Izvestiya, Physics of the Solid Earth, No. 12, 56-70. (In Russian)

[14] Aleshin, A.S. and Pioro, E.V. (2015) O vliyanii obvodnennosti gruntov na resul'taty seismicheskogo mikrorayionirovaniya [On the Influence of Water Content of Soils by Results of Seismic Microzonation]. Injenernye izyskaniya, No. 4, 34-41. (In Russian)

[15] Kriger, N.I., A.S., Kozhevnikov, A.D. and Mindel, I.G. (1994) Seismicheskie svoyistva dispersnych porod [Seismic Properties of Dispersive Grounds]. Injeco, Moscow, 196 p. (In Russian)

[16] Aleshin, A.S. (2015) Osnovy kontinual'noy teorii seismicheskogo mikrorayonirovaniya [Fundamentals of Continuum Theory of Seismic Microzonation]. Earthquake Engineering, Safety of Structures, No. 5, 18-26. (In Russian)

[17] Saks, M.V. and Shteinberg, V.V. (1989) O chastotnoy izbiratel/nosti gruntov pri Spitakskom zemletryasenii 7.12.1988 [About Frequency Selectivity of the Soil Oscillation during the Spitak Earthquake 7.12.1988]. Voprosy Inzhenernoy Seismologii, No. 30, 164-166. (In Russian) 